\title{
Quasiballistic correction to the density of states in three-dimensional metal
}

\author{
A. A. Koulakov \\ Theoretical Physics Institute, University of Minnesota, Minneapolis, Minnesota 55455
}

(December 17, 2013)

\begin{abstract}
We study the exchange correction to the density of states in the three-dimensional metal near the Fermi energy. In the ballistic limit, when the distance to the Fermi level exceeds the inverse transport relaxation time $1 / \tau$, we find the correction linear in the distance from the Fermi level. By a large parameter $\epsilon_{\mathrm{F}} \tau$ this ballistic correction exceeds the diffusive correction obtained earlier.
\end{abstract}

PACS numbers: 71.10.Pm, 73.40.Gk

The zero-bias tunneling anomaly in disordered metals has been studied extensively both experimentally련 and theoretically $\omega^{-1}$. The explanation of this phenomenon has been given on the basis of the interaction induced correction to the single-particle density of states (DOS). For the three-dimensional case the leading order exchange correction is given by:

$$
\frac{\delta \nu_{\text {diff }}}{\nu_{0}}=A \frac{\lambda}{\left(\epsilon_{\mathrm{F}} \tau\right)^{2}} \sqrt{|\varepsilon| \tau}
$$

Here $\nu_{0}=m^{2} v_{\mathrm{F}} / \pi^{2}$ is DOS on the Fermi level in the non-interacting metal ( $m$ and $v_{\mathrm{F}}$ being the mass of electron and the Fermi velocity correspondingly, $\hbar=1$ ), $A=3^{3 / 2} / 8 \sqrt{2} \approx 0.459$ is the numerical constant, and $\lambda$ is the unitless interaction strength. In the realistic cases $\lambda \approx 1$. The subscript attached to the correction to DOS $\delta \nu_{\text {diff }}$ emphasizes that the above result is valid in the diffusive limit, i. e. when the distance to the Fermi level $|\varepsilon|$ is much smaller than the inverse transport scattering time $1 / \tau$. The maximum value that the correction can reach can therefore be estimated as $\delta \nu / \nu \lesssim\left(\epsilon_{\mathrm{F}} \tau\right)^{-2} \ll 1$.

In this work we evaluate the exchange correction to DOS in three-dimensional metal with short-range impurities. The use of the concrete form of disorder allows us to go beyond the universal diffusive regime. Our result in the ballistic regime $(|\varepsilon| \gg 1 / \tau)$ is:

$$
\frac{\delta \nu_{\text {ball }}}{\nu_{0}}=B \frac{\lambda}{\left(\epsilon_{\mathrm{F}} \tau\right)^{2}}|\varepsilon| \tau \text {. }
$$

Here $B=\pi / 16 \approx 0.196$ is the numerical constant, $\lambda=\tilde{V}(\omega=0, \boldsymbol{q}=0) \nu_{0}$, where $\tilde{V}(\omega, \boldsymbol{q})$ is the Fourier transform of screened electron-electron interaction potential. This result is valid up until energies of the order of Fermi energy. The maximum value reached by this correction can be estimated as $\delta \nu / \nu \lesssim\left(\epsilon_{\mathrm{F}} \tau\right)^{-1}$. Thus it exceeds the diffusive correction by a large parameter $\epsilon_{\mathrm{F}} \tau$. We conclude therefore that the ballistic correction produces a larger suppression of DOS on the Fermi level than the diffusive correction in case of short range impurities.

The singular diffusive correction th the tunneling DOS is observed in many experiments. 13 However at larger energies it crosses over into less singular correction, behaving approximately as the absolute value of distance to the Fermi level. This behavior is consistent with our prediction (2) for the ballistic regime. The details of the crossover between (11) and (2) are given below.

To derive Eq. (2) we follow the guidelines of Refs. 11 and 1 . The correction to one particle DOS is related to the exchange correction to the retarded Green function

$$
\delta \nu(\varepsilon)=-\frac{2}{\pi} \int \frac{d \boldsymbol{p}}{(2 \pi)^{3}} \operatorname{Im} \delta G^{R}(\varepsilon, \boldsymbol{p})
$$

The latter is calculated in the first order of the perturbation theory in the electron-electron interaction $\tilde{V}(\omega, \boldsymbol{q})$

$$
\begin{aligned}
& \delta G^{R}(\varepsilon, \boldsymbol{p})=i\left[G^{R}(\varepsilon, \boldsymbol{p})\right]^{2} \int \frac{d \boldsymbol{q}}{(2 \pi)^{3}} \frac{d \omega}{2 \pi} \\
& \times\left[\Gamma(\omega, \boldsymbol{q})^{2}-1\right] G^{A}(\varepsilon-\omega, \boldsymbol{p}-\boldsymbol{q}) \tilde{V}(\omega, \boldsymbol{q}) .
\end{aligned}
$$

Here $G^{R}$ and $G^{A}$ are the retarded and advanced Green functions respectively $G^{R, A}(\boldsymbol{p}, \varepsilon)=$ $1 /\left(\varepsilon-\boldsymbol{p}^{2} / 2 m \pm i / 2 \tau\right)$. We subtract unity from the square of the vertex function in the presence of impurities $\Gamma(\omega, \boldsymbol{q})$ to exclude "bare" interaction correction existing with no impurities. The vertex function has to be calculated using our model of impurity potential

$$
u(\boldsymbol{r})=\sum_{i} u_{0} \delta\left(\boldsymbol{r}-\boldsymbol{r}_{i}\right)
$$

where the locations of impurities $\boldsymbol{r}_{i}$ are scattered randomly with average density $n_{i}$. In the ladder approximation the vertex is then given by

$$
\begin{aligned}
& \Gamma(\omega, \boldsymbol{q})=\theta[\varepsilon(\varepsilon-\omega)]+\frac{\theta(\varepsilon) \theta(\omega-\varepsilon)}{1-\zeta(\omega, \boldsymbol{q})} \\
& +\frac{\theta(-\varepsilon) \theta(\varepsilon-\omega)}{1-\zeta^{*}(\omega, \boldsymbol{q})}
\end{aligned}
$$

where

$$
\begin{aligned}
& \zeta(\omega, \boldsymbol{q})=n_{i}\left|u_{0}\right|^{2} \int \frac{d \boldsymbol{p}}{(2 \pi)^{3}} G^{R}(\boldsymbol{p}+\boldsymbol{q}, \varepsilon) G^{A}(\boldsymbol{p}, \varepsilon-\omega) \\
& =\frac{i}{2 q v_{\mathrm{F}} \tau} \ln \frac{\omega+q v_{\mathrm{F}}+i / \tau}{\omega-q v_{\mathrm{F}}+i / \tau}
\end{aligned}
$$


and $1 / \tau=\pi \nu_{0} n_{i}\left|u_{0}\right|^{2}$. The latter expression is valid for any $\omega, \varepsilon, q v_{\mathrm{F}} \ll \epsilon_{\mathrm{F}}$. In the diffusive case $\left(\omega, \varepsilon, q v_{\mathrm{F}} \ll\right.$ $1 / \tau)$ the last expression takes the form $\zeta=1+i \omega \tau-$ $\left(q v_{\mathrm{F}} \tau\right)^{2} / 3$ and the vertex part has the expected diffusive pole.

The screened electron-electron interaction $\tilde{V}(\omega, \boldsymbol{q})=$ $4 \pi e^{2} /\left[q^{2}+4 \pi e^{2} \Pi(\omega, \boldsymbol{q})\right]$, where the polarization operator derived in the random phase approximation

$$
\Pi(\omega, \boldsymbol{q})=\nu_{0}[1+\alpha i \omega \tau \zeta /(1-\zeta)]
$$

Here parameter $\alpha$ is equal to 1 or 0 depending on whether the retardation of the interaction is to be taken into account or not. This parameter is introduced for comparison to the earlier results.

After some transformations the expression for the correction to DOS is obtained from Eq. (4)

$$
\frac{\delta \nu}{\nu_{0}}=\frac{\lambda}{\left(\epsilon_{\mathrm{F}} \tau\right)^{2}} f(|\varepsilon| \tau),
$$

where the interaction constant $\lambda=1$ and

$$
\begin{aligned}
& f(\gamma)=\frac{1}{8 \pi} \int_{0}^{\gamma} d \gamma^{\prime} \operatorname{Im} \int_{0}^{\infty} \frac{x^{2} d x}{x^{2}-\left(\gamma^{\prime}+i\right)^{2}} \\
& \times \frac{2 \zeta-\zeta^{2}}{(1-\zeta)\left[1-\zeta\left(1-\alpha i \gamma^{\prime}\right)\right]}, \\
& \zeta=\frac{i}{2 x} \ln \frac{\gamma^{\prime}+x+i}{\gamma^{\prime}-x+i} .
\end{aligned}
$$

The integrals in (10) cannot be evaluated in terms of elementary functions. Nevertheless the asymptotic expressions for diffusive $(\gamma \ll 1)$ and ballistic $(\gamma \gg 1)$ cases are easily obtained:

$$
f(\gamma)= \begin{cases}A \sqrt{\gamma}, & \gamma \ll 1 \\ B \gamma, & \gamma \gg 1\end{cases}
$$

They lead to Eqs. (11) and (2). The constant $A$ in this formula differs for the cases of instantaneous $(\alpha=0)$ and retarded $(\alpha=1)$ interactions. For the former case we obtain $A=3^{3 / 2} / 16 \sqrt{2}$, for the latfer $A=3^{3 / 2} / 8 \sqrt{2}$. This agrees with the previous results. 1 The ballistic constant $B=\pi / 16$ is the same for both instantaneous and retarded cases.

The crossover between diffusive and ballistic regimes can be described numerically. To this end we represent the correction to DOS in the intermediate region as follows

$$
\delta \nu=C \sqrt{\delta \nu_{\mathrm{diff}}^{2}+\delta \nu_{\mathrm{ball}}^{2}}
$$

where the crossover function $C \approx 1$ is shown in Fig. 11.

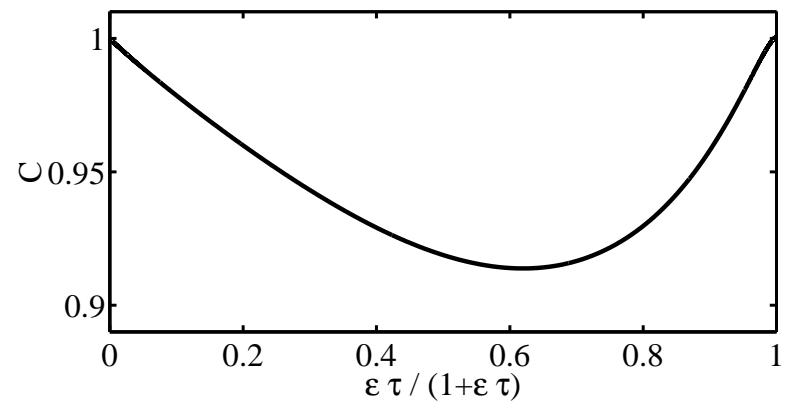

FIG. 1. The crossover function.

The crossover function can be effectively fitted with polynomials

$$
C(\xi)=\sum_{n} C_{n} \xi^{n}, \xi \equiv \varepsilon \tau /(1+\varepsilon \tau)
$$

The fit with a 7 th degree polynomial with coefficients $C_{0}=0.9999, C_{1}=-0.2610, C_{2}=0.9114, C_{3}=-5.6062$, $C_{4}=17.9270, C_{5}=-28.7905, C_{6}=22.8587$, and $C_{7}=-7.0387$ guarantees an error not exceeding $0.14 \%$.

In conclusion we evaluated the exchange correction to the tunneling density of states in three-dimensional metal in the ballistic regime $\left(1 / \tau \ll \varepsilon \ll \epsilon_{\mathrm{F}}\right)$. The obtained correction is proportional to the distance to the Fermi level $\varepsilon$ and exceeds the diffusive correction by the large parameter $\epsilon_{\mathrm{F}} \tau$. The crossover between diffusive and ballistic limits is also studied.

The author is grateful to Boris Shklovskii and Alexander Rudin for valuable discussions. This work is supported by NSF grant DMR-9616880.

${ }^{1}$ B. L. Altshuler and A. G. Aronov, In: "Electron-electron interaction in disordered conductors", Edited by A. L. Efros and M. Pollak, Elsevier, 1985, p. 1.

${ }^{2}$ W. L. McMillan and J. Mochel, Phys. Rev. Lett. 46, 556 (1981); Y. Imry and Z. Ovadyahu, Phys. Rev. Lett. 49, 841 (1982); G. Hertel, D. J. Bishop, E. G. Spencer, J. M. Rowell, and R. C. Dynes, Phys. Rev. Lett. 50, 743 (1983); A. E. White, R. C. Dynes, and J. P. Garno, Phys. Rev. B 31, 1174 (1985); Phys. Rev. Lett. 56, 532 (1986); J. G. Massey and Mark Lee, Phys. Rev. Lett. 77, 3399 (1996).

${ }^{3}$ M. Lee, J. C. Massey , V. L. Nguyen , B. I. Shklovskii , Phys. Rev. B 601582 (1999).

${ }^{4}$ A. M. Rudin, I. L. Aleiner, L. I. Glazman, Phys. Rev. B 55, 9322 (1997). 